\title{
Escala de Pensamentos Automáticos para Crianças e Adolescentes (EAP): adaptação e propriedades psicométricas
}

\author{
Maycoln Teodoro - Universidade Federal de Minas Gerais, Minas Gerais, Brasil \\ Aline Abreu e Andrade - Universidade Federal de Minas Gerais, Minas Gerais, Brasil \\ Heitor Amâncio de Moraes Castro - Universidade Federal de Minas Gerais, Minas Gerais, Brasil
}

\begin{abstract}
Resumo
O objetivo deste estudo foi adaptar e investigar algumas propriedades psicométricas da Escala de Pensamentos Automáticos para Crianças e Adolescentes (EPA) (Children's Automatic Thoughts Scale, CATS). Foram avaliadas 326 crianças e adolescentes, sendo 135 meninos (41,40\%) e 189 meninas (58,00\%) com idade variando entre nove e 16 anos (Média=12,34, DP=1,48 anos). Os participantes responderam à EPA e ao Inventário de Depressão Infantil. Os resultados indicaram uma estrutura fatorial com quatro fatores para a EPA semelhantes à escala original, bem como consistência interna satisfatória para todos os agrupamentos. Foram encontradas correlações significativas entre a intensidade dos pensamentos automáticos disfuncionais e os sintomas de depressão, sendo que o fator fracasso pessoal mostrou-se um preditor significativo da intensidade da depressão. Os resultados iniciais sugerem ser a EPA um instrumento promissor a ser adotado no Brasil para a avaliação dos pensamentos automáticos de crianças e adolescentes. Palavras-chave: Terapia cognitiva, Escalas, Depressão, Crianças, Adolescentes.
\end{abstract}

\section{Children's Automatic Thoughts Scale (Cats): adaptation and psychometric properties}

\begin{abstract}
The objective of this study was to adapt and investigate the psychometric properties of the Childrens Automatic Thoughts Scale (CATS). The sample was composed by 326 children and adolescents, 135 boys (41.40\%) and 189 girls $(58.00 \%)$. Ages ranged between 9 and 16 years old (Mean=12.34, SD=1.48 years). The respondents answered the CATS and the Child Depression Inventory. The results indicated a factorial structure with four factors for the CATS similar to the original scale, as well as satisfactory internal consistency. Significant correlations were found between the intensity of the automatic dysfunctional thoughts and the depression symptoms. The factor personal failure was a significant predictor of depression intensity. Initial results suggest that the CATS is a promising tool to be adopted in Brazil for assessing children's and adolescents' automatic thoughts.

Key words: Cognitive therapy, Scales, Depression, Children, Adolescents.
\end{abstract}

\section{Escala de Pensamientos Automáticos para niños y adolescentes (EPA): adaptación y propiedades} psicométricas

\begin{abstract}
Resumen
El objetivo de este estudio fue adaptar e investigar algunas propiedades psicométricas de la Escala de Pensamientos Automáticos para Niños y Adolescentes (EPA) (Children's Automatic Thoughts Scale, CATS). Fueron evaluados 326 niños y adolescentes, siendo 135 niños $(41,40 \%)$ y 189 niñas $(58,00 \%)$ con edad variando entre nueve y 16 años (Promedio=12,34, DP=1,48 años). Los participantes respondieron a la EPA y al Inventario de Depresión Infantil. Los resultados indicaron una estructura con cuatro factores para la EPA semejantes a la escala original, bien como consistencia interna satisfactoria para todos los agrupamientos. Fueron encontradas correlaciones significativas entre la intensidad de los pensamientos automáticos disfuncionales y los síntomas de depresión. Los resultados iniciales sugieren que la EPA es un instrumento prometedor a ser adoptado en Brasil para la evaluación de los pensamientos automáticos de niños y adolescentes.

Palabras clave: Terapia cognitiva, Escalas, Depresión, Niños, Adolescentes.
\end{abstract}

As teorias embasadas pelo modelo da terapia cognitiva postulam que o comportamento $\mathrm{e}$ as emoções das pessoas são modulados pela interpretação das situações e não pelas situações em si. Haveria, portanto, um componente cognitivo mediador entre os estímulos ambientais e as respostas emitidas pelo sujeito, sejam emoções, pensamentos, comportamentos ou reações fisiológicas. Essa concepção não é totalmente nova e já estava presente entre os filósofos estoicos, como Epíteto e Marco Aurélio (Beck, Rush, Shaw, \& Emery, 1997; Dobson \& Dozois, 2006).

Segundo Beck (2011), a significação dos eventos se dá mediante três estruturas mentais

Disponivel em www.scielo.br interdependentes. A primeira é composta pelo conjunto de crenças centrais, delineadas pelas concepções mais profundas e estáveis que um indivíduo tem sobre si, sobre o futuro e sobre o mundo que o rodeia. A segunda, desdobrando-se da primeira e retroalimentando-a, é formada pelas crenças intermediárias. São ideias mais específicas e maleáveis, que influenciam fortemente os pensamentos, sentimentos e ações do indivíduo, e incluem atitudes, regras e suposições, geralmente seguindo um formato "se... então..." (por exemplo, "se não for eficaz sempre, então serei um fracassado”). A última estrutura é denominada de pensamento automático (PA) e surge 
segundo a ativação das crenças mais profundas em função de um dado contexto. Os PAs são mais superficiais e aparecem rapidamente de forma semiconsciente à medida que uma situação é experimentada e interpretada, deixando como rastro um efeito emocional. Normalmente as pessoas não se dão conta de sua existência ou de seu conteúdo, mas os mesmos podem se tornar conscientes por meio de um processo atencional.

Por afetarem tão diretamente a emoção, os pensamentos automáticos estão intrinsecamente relacionados à saúde. Pensamentos automáticos causados por crenças centrais e intermediárias desadaptativas geram emoções adversas. Nesse sentido, diversos transtornos psicológicos seguem um padrão cognitivo peculiar, ou seja, são mantidos por certas crenças centrais disfuncionais que acabam por desencadear pensamentos automáticos que comprometem a qualidade de vida (Gabbard, Beck \& Holmes, 2007). Por exemplo, Beck e Alford (2011) afirmam que os sintomas depressivos estão relacionados com a ativação de três padrões cognitivos, chamados de tríade cognitiva, que conduzem o indivíduo a ter uma visão negativa do si mesmo (self), do mundo e do futuro.

A detecção da cognição disfuncional, que constitui o substrato dos distúrbios psicológicos de acordo com o modelo da terapia cognitiva (Beck, 2011), pode ser feita de várias maneiras. Além da entrevista terapêutica e de algumas técnicas específicas, existe a possibilidade de se avaliar a ocorrência e intensidade dos pensamentos automáticos por meio de instrumentos psicológicos. O uso de escalas pode ser especialmente útil quando se trata da investigação das cognições de crianças e adolescentes, tendo em vista a facilidade existente no processo de reconhecimento das crenças. Nesse contexto, algumas escalas vêm sendo criadas no intuito de discriminar padrões disfuncionais que podem ser preditivos para distintos distúrbios psicológicos, como depressão e transtorno de ansiedade na infância e adolescência. Um exemplo é o Children's Cognitive Assessment Questionnaire (CCAQ; Zatz \& Chassin, 1983), um questionário que mede pensamentos automáticos relacionados à ansiedade. Além disso, o desenvolvimento desses instrumentos tem se mostrado útil para embasar empiricamente os modelos teóricos relacionados aos diversos quadros que explicam a dinâmica não apenas dos transtornos psicológicos, mas do funcionamento cognitivo de forma geral.

São encontrados atualmente, na literatura, alguns instrumentos específicos para a mensuração de pensamentos automáticos, dentre eles o Automatic Thoughts Questionnaire (ATQ-30; Hollon \& Kendall,
1980). O ATQ-30 foi uma das primeiras escalas desenvolvidas para identificar e medir a frequência de ocorrência de pensamentos automáticos negativos relacionados ao self. Esse questionário é composto por 30 itens de autorrelato, que devem ser respondidos por meio de uma escala Likert de cinco pontos, divididos em quatro fatores (desajustamento pessoal, autoconceito e expectativa negativa, baixa autoestima e desesperança). Hollon e Kendall encontraram boa discriminação entre grupos não-clínicos e pacientes deprimidos e consistência interna adequada para os fatores. Posteriormente, no intuito de avaliar também as cognições positivas, Kendall, Howard e Hays (1989) incluíram dez itens no ATQ-30 original, criando o ATQ-R.

A intenção de avaliar os pensamentos automáticos positivos também foi o princípio norteador no desenvolvimento da versão do ATQ-P (Ingram \& Wisnicki, 1988). Baseando-se na ideia de Hollon e Kendall (1980) no desenvolvimento do ATQ-30, os autores construíram uma versão com 30 itens positivos distribuídos em quatro fatores (funcionamento diário positivo, autoavaliação positiva, outras avaliações do self e expectativas futuras positivas). As propriedades psicométricas do ATQ-P foram satisfatórias, com boa discriminação entre grupos clínicos (ver Ingram, Kendall, Siegle, Guarino, \& McLaughlin, 1995, para uma revisão).

Outro questionário que investiga os pensamentos automáticos é o Negative Affect Self-Statement Questionnaire (NASSQ, Ronan, Kendall, \& Rowe, 1994). O NASSQ foi desenvolvido para avaliar afirmações associadas com afetos negativos em crianças e adolescentes. A escala possui 53 itens, divididos em duas subescalas. A primeira, com 14 itens, é destinada a crianças de sete a dez anos e a segunda, com 39, a adolescentes de 11 a 15 anos. A primeira subescala possui itens divididos em dois fatores, dos quais um relacionado a aspectos depressivos e outro a ansiedade. A segunda subescala possui, além desses dois fatores, um terceiro, com itens que descrevem o afeto negativo. Os autores encontraram índices de consistência interna satisfatórios e correlação significativa dos fatores com escalas de depressão e ansiedade.

Uma escala de avaliação dos pensamentos automáticos que vem sendo muito estudada e descrita na literatura atualmente é a Children's Automatic Thoughts Scale (CATS, Schniering \& Rapee, 2002). Desenvolvida na Austrália, a CATS investiga pensamentos automáticos em crianças e adolescentes por meio de 40 itens que devem ser respondidos em uma escala Likert de cinco pontos (variando de nunca até todo o tempo). Todas as sentenças possuem o formato de autorrelato e são divididas em quatro fatores. O primeiro é chamado 
de ameaça social e contém itens relativos ao medo da não-aceitação social. O segundo, ameaça física, possui frases com conteúdo de preocupações com acidentes, enquanto o terceiro, fracasso pessoal, possui ideias de desvalorização pessoal. O último fator, hostilidade, está relacionado ao sentimento de vingança. Estudos anteriores têm investigado amostras com idade entre sete e 17 anos e encontrado índices de consistência interna satisfatórios (superiores a 0,82) e boa estabilidade teste-reteste $(r=0,91)$ (Schniering \& Rapee, 2002, 2004b; Schniering \& Lyneham, 2007).

A CATS permite acessar pensamentos automáticos relacionados a sintomas internalizantes e externalizantes de crianças e adolescentes. Possui também uma boa capacidade de discriminar entre grupos clinicamente diagnosticados e grupos nãoclínicos, especialmente em crianças e adolescentes com transtorno de ansiedade, fobia ou depressão (Schniering \& Rapee, 2004ª; Schniering \& Lyneham, 2007; Micco \& Ehrenreich, 2009; Hogendoorn, Wolters, Vervoort, Prins, Boer, Kooij, \& Haan, 2010).

Tendo em vista a importância da avaliação dos pensamentos automáticos tanto para a prática clínica quanto para a pesquisa, este estudo teve por finalidade adaptar para o português a Children's Automatic Thoughts Scale (CATS), chamada de Escala de Pensamentos Automáticos para Crianças e Adolescentes (EPA) e investigar algumas de suas propriedades psicométricas Foram analisadas a estrutura fatorial e a consistência interna dos itens, assim como a sua relação com os sintomas depressivos de crianças e adolescentes.

\section{Método}

\section{Participantes}

Participaram deste estudo 326 crianças e adolescentes de escolas públicas (83, 25,50\%) e particulares $(243,74,50 \%)$ da cidade de Belo Horizonte, Minas Gerais. A amostra foi composta por 189 estudantes do sexo feminino $(58,00 \%)$ e 135 do sexo masculino $(41,40 \%)$. A idade variou de nove a 16 anos (Média $=12,34, \mathrm{DP}=1,48$ anos). Dois participantes $(0,60 \%)$ não informaram o sexo e a idade. Uma subamostra participou do estudo de validade concorrente e contou com 124 crianças e adolescentes, sendo 81 meninas $(65,30 \%)$ e 43 meninos $(34,70 \%)$. A idade desse subgrupo variou de dez a 16 anos (Média=12,30, DP=1,60 anos). $\mathrm{O}$ critério de seleção amostral utilizado foi o de conveniência.

\section{Instrumentos}

Escala de Pensamentos Automáticos para Crianças e Adolescentes (EPA, Children's Automatic Thoughts Scale)
A EPA (Schniering \& Rapee, 2002) investiga pensamentos automáticos em crianças e adolescentes por meio de 40 itens, que devem ser respondidos em uma escala Likert de cinco pontos $(0$ a 4 , variando de nunca até todo o tempo). Os itens expressam emoções negativas que podem ser exploradas em diversos tipos de transtornos internalizantes e externalizantes. Todas as sentenças possuem o formato de autorrelato e são divididas em quatro fatores, com dez itens cada, que avaliam a ameaça social, ameaça física, falha pessoal e hostilidade. Estudos anteriores, que investigaram amostras com idade entre sete a 17 anos, apresentaram consistência interna adequada (superior a 0,82 ) e boa estabilidade teste-reteste $(\mathrm{r}=0,91)$. Além disso, o instrumento apresenta resultados discriminantes entre subamostras clínicas e controles (Schniering \& Rapee, 2002; Schniering \& Lyneham, 2007).

Inventário de Depressão Infantil (CDI)

O Inventário de Depressão Infantil (CDI, Children's Depression Inventory, Kovacs, 1992, 2003) possui 27 itens que investigam a intensidade dos sintomas de depressão em crianças e adolescentes. Os participantes precisam escolher a alternativa que melhor descreve o seu estado emocional na atualidade, por meio de uma escala Likert de três pontos. A adaptação do CDI para o Brasil foi feita por Gouveia, Barbosa, Almeida e Gaião (1995) em um estudo realizado com 305 estudantes de João Pessoa e apresentou consistência interna de 0,81. Em outras investigações, Wathier, Dell'Aglio e Bandeira (2008) encontraram um alfa de 0,85 em uma amostra de 951 crianças e adolescentes em Porto Alegre, enquanto os resultados de Cruvinel, Boruchovitch e Santos (2008) tiveram um escore de 0,80 . Considerando a subamostra que preencheu o CDI no presente estudo, foi encontrado um alfa de Crombach de 0,85 para a escala.

\section{Procedimentos de pesquisa e éticos}

A tradução da Children's Automatic Thoughts Scale (CATS) para o português foi autorizada pelos autores e realizada por três juízes, que produziram, independentemente, três versões da escala. Essas traduções foram comparadas e discutidas por um comitê de quatro juízes, que formularam uma versão inicial da Escala de Pensamentos Automáticos. Essa versão foi traduzida para o inglês e comparada com a CATS original, resultando na EPA final.

A aplicação da EPA e do CDI nos alunos das escolas públicas e particulares foi feita coletivamente e dentro da sala de aula. $O$ contato inicial foi estabelecido com a direção da escola. Após a permissão, foi explicado para as crianças e adolescentes o objetivo da pesquisa e solicitado que os mesmos 
levassem o Termo de Consentimento Livre e Esclarecido para ser assinado pelos pais ou responsáveis. O projeto foi aprovado pelo comitê de ética da universidade dos autores.

\section{Análise dos dados}

A investigação das propriedades psicométricas da EPA foi realizada por meio de análise fatorial exploratória e de consistência interna (alfa de Crombach). As comparações entre grupos foram realizadas com o Teste $\mathrm{t} e$ as associações foram feitas por meio de correlação de Pearson e análise de regressão linear. Todas as variáveis apresentaram distribuição normal segundo o Teste de KolmogorovSmirnov. A análise estatística foi realizada no Programa Estatístico SPSS 16.

\section{Resultados}

A análise fatorial exploratória foi realizada com método dos Eixos Principais (Principal Axis Factoring) e rotação promax. A solução para quatro fatores foi escolhida por ser a estrutura original do instrumento e pela análise do Gráfico de Sedimentação. Os resultados podem ser visualizados na Tabela 1.

Tabela 1. Cargas fatoriais, variância explicada e alfas de Crombach para os escores da Escala de Pensamentos Automáticos para Crianças e Adolescentes (EPA) $(n=326)$ (continua)

\begin{tabular}{|c|c|c|c|c|}
\hline \multirow[t]{2}{*}{ Itens } & \multicolumn{4}{|c|}{ Fatores } \\
\hline & $\begin{array}{c}\text { Fracasso } \\
\text { pessoal }\end{array}$ & $\begin{array}{c}\text { Ameaça } \\
\text { social }\end{array}$ & $\begin{array}{c}\text { Ameaça } \\
\text { física }\end{array}$ & Hostilidade \\
\hline 38. Eu me odeio. & 0,92 & $-0,20$ & & \\
\hline 28. Eu sou um fracasso. & 0,84 & & & $-0,21$ \\
\hline 11. Eu não sirvo pra nada. & 0,77 & & & \\
\hline 13. Nada mais dá certo pra mim. & 0,73 & & & \\
\hline 23. Eu transformei minha vida em uma grande bagunça. & 0,71 & & & \\
\hline 25. Eu pareço um idiota. (AS) & 0,62 & & & \\
\hline 36. Tem alguma coisa muito errada comigo. (AF) & 0,51 & 0,25 & & \\
\hline 26. Eu nunca serei tão bom quanto as outras pessoas. & 0,50 & & & \\
\hline 03. Eu não consigo fazer nada direito. & 0,46 & & & \\
\hline 27. Eu sempre levo a culpa por coisas que eu não faço. $(\mathrm{H})$ & 0,42 & & & \\
\hline 34. Eu nunca vou superar meus problemas. & 0,41 & & & \\
\hline 07. Eu estou ficando louco. (AF) & 0,40 & & & \\
\hline 17. É minha culpa se as coisas deram errado. & 0,36 & 0,27 & & \\
\hline 14. Eu vou parecer um bobão. (AS) & 0,33 & 0,28 & & \\
\hline 10. A maioria das pessoas está contra mim. $(\mathrm{H})$ & 0,30 & 0,28 & & \\
\hline 06. Fico com medo de ser gozado. & & 0,72 & $-0,24$ & \\
\hline 21. Eu tenho medo do que as outras crianças vão pensar sobre mim. & & 0,69 & & \\
\hline 32. Eu tenho medo de fazer papel de bobo. & & 0,67 & & \\
\hline 08. As outras crianças vão rir de mim. & & 0,65 & & \\
\hline 29. As outras crianças zombam de mim. & & 0,64 & & \\
\hline 01. As outras crianças vão pensar que eu sou burro. & & 0,42 & & \\
\hline 18. As outras pessoas pensam coisas ruins sobre mim. & & 0,41 & & \\
\hline 16. Tenho medo de perder o controle (AF) & & 0,39 & & \\
\hline 31. Todos estão me encarando. & & 0,39 & & \\
\hline 35. As pessoas sempre tentam me meter em confusão. $(\mathrm{H})$ & & 0,30 & & 0,24 \\
\hline 39. Alguma coisa vai acontecer com alguém que eu me preocupo. & & & 0,72 & \\
\hline 20. Eu vou me machucar. & & & 0,67 & \\
\hline 09. Eu vou morrer. & & & 0,63 & \\
\hline
\end{tabular}


Tabela 1. Cargas fatoriais, variância explicada e alfas de Crombach para os escores da Escala de Pensamentos Automáticos para Crianças e Adolescentes (EPA) $(n=326)$

Itens
(continuação)

Fatores

Fracasso Ameaça Ameaça Hostilidade pessoal social física

04. Eu vou sofrer um acidente.

24. Algo de muito ruim vai acontecer.

12. Minha mãe ou meu pai vão se machucar.

30. Não vale a pena viver. (FP)

33. Tenho medo de que alguém possa morrer.

19. Se alguém me machucar eu tenho o direito de machucá-los de volta.

02. Eu tenho o direito de me vingar das pessoas, se elas merecerem

15. Eu não vou deixar barato quando alguém me incomodar.

22. Algumas pessoas merecem o que acontece com elas.

40. Pessoas más merecem ser punidas.

05. As outras crianças são burras.

37. Algumas pessoas são más.
Variância Explicada

Alfa de Crombach

$\begin{array}{ccc} & -0,21 & 0,62 \\ 0,33 & & 0,57 \\ & 0,23 & 0,45 \\ 0,37 & & 0,43 \\ & & 0,39\end{array}$

0,62

0,57

0,43

0,39

0,73

0,71

0,64

$0,28 \quad 0,51$

0,47

0,47

0,26

Nota: Análise fatorial com o método dos eixos principais (Principal Axis Factoring) e rotação promax. Somente as cargas fatoriais maiores do que 0,20 foram apresentadas. As siglas entre parênteses representam o fator original do item no artigo de Schniering e Rapee (2002). (AS): Ameaça Social; (AF): Ameaça Física; (H): Hostilidade; (FP): Fracasso Pessoal.

A análise fatorial com quatro fatores apresentou escore de Kaiser-Meyer-Olkin de 0,93 e teste de Esfericidade de Bartlett significativo, indicando adequação desta estrutura para a amostra estudada. A solução fatorial final explicou $40,86 \%$ da variância, conforme distribuição apresentada na Tabela 1.

O primeiro fator, fracasso pessoal, ficou com 15 itens, dos quais seis pertenciam a outras dimensões na escala original. O segundo, ameaça social, possui dez itens, dos quais dois pertenciam a outros fatores originalmente. $\mathrm{O}$ fator ameaça física possui oito itens, sendo que um não pertencia a essa subescala na versão original. Finalmente, o fator hostilidade possui sete itens, sendo que todos pertenciam a essa mesma dimensão no estudo de Schniering e Rapee (2002). Um item (algumas pessoas são más) não alcançou carga fatorial superior a 0,30 , mas foi mantido no fator hostilidade.

A partir da análise dos itens e seu respectivo agrupamento (vide Tabela 1), pode-se caracterizar altos escores no fator 1, fracasso pessoal, como indicador de altos índices de pensamentos que expressam desvalorização pessoal com expressão de culpa e de inutilidade. A pontuação alta em ameaça social mostra ideias de desvalorização social, principalmente aquelas relacionadas a um pior rendimento, quando comparados com pares e medo relativos à aceitação social. O fator ameaça física contém itens que expressam preocupação com acidentes e mortes de familiares ou da própria criança e adolescente. Altos escores no fator 4, hostilidade, representam um alto número de pensamentos automáticos relacionados à vingança pessoal e desvalorização de outras pessoas.

Análises de correlações entre os fatores da EPA mostraram escores significativos entre todas as relações. Fracasso pessoal correlaciona-se positivamente com hostilidade $(\mathrm{r}=0,38, \mathrm{p}<0,001)$, ameaça física $(\mathrm{r}=0,66, \mathrm{p}<0,001)$ e ameaça social $(\mathrm{r}=0,73, \mathrm{p}<0,001)$. Hostilidade, por sua vez, possui correlação positiva com ameaça física $(\mathrm{r}=0,36$, $\mathrm{p}<0,001)$ e ameaça social $(\mathrm{r}=0,32, \mathrm{p}<0,001)$. Da mesma forma, os fatores relacionados às ameaças física e social estão positivamente correlacionados $(\mathrm{r}=0,59$, $\mathrm{p}<0,001)$.

As análises de consistência interna foram feitas por meio do alfa de Crombach, e os resultados estão descritos na Tabela 1. Todos os escores encontrados são adequados, sendo 0,91 para fracasso pessoal, 0,85 para ameaça social, 0,81 para ameaça física e 0,76 para hostilidade. $\mathrm{O}$ alfa total da escala foi de 0,94 .

Os itens de cada fator foram somados, originando os escores de cada dimensão conforme mostrado na 
Tabela 1 e o total de pensamentos automáticos mediana estão descritos na Tabela 2. disfuncionais da EPA. A média, desvio padrão e

Tabela 2. Média (desvio padrão), mediana e percentil 75 dos fatores e soma total da Escala de Pensamentos Automáticos para Crianças e Adolescentes (EPA), de acordo com o sexo do participante $(\mathrm{n}=326)$

\begin{tabular}{|c|c|c|c|c|c|c|}
\hline \multirow{2}{*}{ Fator } & \multicolumn{2}{|c|}{ Média (DP) } & \multicolumn{2}{|c|}{ Mediana } & \multicolumn{2}{|c|}{ Percentil 75} \\
\hline & M & $\mathrm{F}$ & $\mathrm{M}$ & $\mathrm{F}$ & $\mathrm{M}$ & $\mathrm{F}$ \\
\hline Fracasso Pessoal & $12,49(11,86)$ & $14,02(11,63)$ & 9,00 & 10,00 & 17,00 & 20,00 \\
\hline Ameaça Social & $9,48(7,49)$ & $10,52(7,64)$ & 8,00 & 9,00 & 14,00 & 14,00 \\
\hline Ameaça Física & $6,70(6,06)$ & $6,34(5,94)$ & 5,00 & 5,00 & 9,00 & 9,00 \\
\hline Hostilidade & $11,81(6,06)$ & $9,62(5,53)$ & 11,00 & 9,00 & 16,00 & 13,00 \\
\hline EPA Total & $40,48(24,89)$ & $40,51(25,91)$ & 34,00 & 34,00 & 54,00 & 59,00 \\
\hline
\end{tabular}

Com base nas definições dos fatores, foram realizadas análises de correlação entre os escores da EPA e a idade dos participantes, nenhum resultado tendo sido significativo. Da mesma forma, não foi encontrada diferença significativa entre alunos oriundos de escolas públicas e particulares. Comparações entre os grupos masculino e feminino indicaram que a intensidade de pensamentos com o conteúdo de hostilidade (fator 4) é superior em meninos $(M=11,81, D P=6,06)$ quando comparada com a das meninas $(M=9,62, D P=5,53)(t=3,37, p<0,001)$.

As associações entre as intensidades dos pensamentos automáticos disfuncionais avaliados pela EPA e dos sintomas de depressão (CDI) indicaram correlações significativas e positivas em todas as análises. A intensidade da depressão correlacionou-se com o fracasso pessoal $(r=0,76, p<0,001)$, a hostilidade $(r=0,32, p<0,001)$, a ameaça física $(r=0,46, p<0,001)$, a ameaça social $(r=0,52, p<0,001)$ e a soma total da EPA $(r=0,69, p<0,001)$.

Com o objetivo de testar a predição dos pensamentos automáticos disfuncionais e a intensidade da depressão, foi realizada uma análise de regressão linear múltipla com o método Enter, tendo como variável dependente o escore total do CDI e como preditores os fatores da EPA e o sexo do participante. $\mathrm{A}$ análise mostrou-se adequada $(\mathrm{F}=32,75, p<0,001)$ e explicou $58 \%$ da variância da depressão. Dentre os preditores, foi significativo o fracasso pessoal $(\beta=0,76$, $t=8,18, p<0,001)$. Os demais fatores e o sexo não contribuíram de maneira significativa para a intensidade da depressão.

\section{Discussão}

A identificação dos pensamentos automáticos consiste em uma etapa vital para a implementação da terapia cognitiva. Por isso, desenvolver e adaptar métodos que auxiliem na avaliação dessa estrutura cognitiva possui um forte impacto tanto na clínica quanto na pesquisa em psicoterapia cognitiva. Nesse contexto, a tradução e a adaptação da Escala de Pensamentos Automáticos para Crianças e Adolescentes irá suprir uma lacuna existente na literatura brasileira.

A solução fatorial encontrada para a versão adaptada é semelhante à original em inglês, com exceção de nove itens, que apresentaram carga fatorial mais alta em um fator que não era o de origem. No entanto, esses itens não confundiram a interpretação do fator e, por isso, decidiu-se manter os mesmos nomes dos fatores originais. Dos itens que mudaram de fator, seis estão no primeiro agrupamento, fracasso pessoal, e expressam a ideia de desvalorização pessoal. São eles "Eu pareço um idiota", "Eu vou parecer um bobão" (originalmente ameaça social); "Tem alguma coisa muito errada comigo" e "Eu estou ficando louco" (originalmente ameaça física); "Eu sempre levo a culpa por coisas que eu não faço" e "A maioria das pessoas está contra mim" (hostilidade no original). Uma análise do conteúdo destes itens indica claramente a presença de deficiência pessoal, o que justifica sua inclusão nesse fator. $\mathrm{O}$ segundo fator, ameaça social, possui dois itens ("Tenho medo de perder o controle" e "As pessoas sempre tentam me meter em confusão"), que originalmente pertenciam aos fatores ameaça física $\mathrm{e}$ hostilidade. Ambos foram interpretados como pensamentos negativos vinculados a um contexto social no qual ou a pessoal perderia o controle ou estaria com problemas. O último item, "Não vale a pena viver", classificado como fracasso pessoal na versão australiana, possui carga fatorial no fator ameaça física, na versão brasileira. Essa classificação mostra que os estudantes do presente estudo podem ter interpretado o conteúdo desse item como relacionado a ideações suicidas. $O$ fator hostilidade não apresentou nenhum item advindo de outro grupo. Entretanto, um deles (“Algumas pessoas são más") ficou com a carga fatorial um pouco abaixo das demais $(0,26)$. No entanto, decidiu-se por manter esse item e conservar a quantidade de frases do instrumento em inglês. 
A estrutura fatorial da EPA explicou 40,86\% da variância e apresentou escores no alfa de Cronmbach variando de 0,76 a 0,91 para os fatores. Esses resultados são adequados e estão próximos aos valores originalmente encontrados por Schniering e Rapee (2002, 2004b) e Schniering e Lyneham (2007).

Neste estudo não foi encontrada nenhuma diferença em relação dos fatores da EPA com a idade do participante (amostra entre nove e 16 anos). A ausência de correlações significativas é apoiada pelos achados de Schniering e Rapee (2002, 2004b) em uma amostra não-clínica de crianças e adolescentes de sete a 16 anos. Todos esses resultados são interessantes quando comparados aos de outro estudo com crianças diagnosticadas com algum transtorno de ansiedade e idade variando de sete a 17 anos (Schniering \& Lyneham, 2007). Nessa pesquisa, foi encontrada uma elevação da intensidade no fator ameaça social tanto para meninos quanto para meninas com o aumento da idade. Segundo Schniering e Lyneham, essa diferença é consistente com os achados que mostram uma maior prevalência de fobia social e depressão na adolescência. Todo esse conjunto de dados está de acordo com o teorizado por Beck (2011), que diz que a consolidação das crenças cognitivas ocorreria na adolescência. Nesse caso, o desenvolvimento das crenças cognitivas na infância seguiria o mesmo padrão na adolescência na ausência de psicopatologia. No entanto, destaca-se que esse estudo é transversal e que essa explicação precisa ser confirmada por estudos longitudinais.

Os dados encontrados na literatura sobre diferenças sexuais em pensamentos automáticos são discordantes. Comparações entre o sexo dos participantes no presente estudo sugerem que os meninos possuem uma frequência maior de pensamentos hostis (fator 4, hostilidade) do que as meninas. Com relação aos outros fatores, não foi encontrada nenhuma diferença significativa entre os sexos dos participantes. Schniering e Rapee (2002) e Hogendoorn e cols. (2010) também encontraram uma maior intensidade de hostilidade entre meninos. Por outro lado, Schniering e Rapee (2002) verificaram que as meninas possuem escores superiores aos meninos em fracasso pessoal (fator 1) e ameaça social (fator 2), enquanto o estudo de Schniering e Rapee (2004b) não mostrou nenhuma diferença entre os grupos. Os altos escores de hostilidade em grupos de meninos, encontrados em três estudos, podem estar vinculados à maior presença de fatores externalizantes em crianças e adolescentes desse sexo, como também ao fato de que os meninos são mais expostos à hostilidade familiar do que as meninas (Rutter, 1996), o que poderia gerar uma maior aprendizagem dessa crença.
As correlações entre os quatro fatores e a escala total da EPA e a intensidade dos sintomas de depressão avaliados pelo CDI mostraram associações positivas e significativas que variaram de 0,32 a 0,76. Esses achados são apoiados pelos resultados de Schniering e Rapee (2004a), que encontraram correlações variando de 0,48 a 0,71 e Schniering e Lyneham (2007), com escores variando de 0,43 a 0,76 , ambos comparando os pensamentos automáticos com o CDI. Tanto no presente trabalho quanto nas investigações realizadas por Schniering e colaboradores, o maior índice de correlação era com o fator fracasso pessoal e o menor, com a hostilidade. Utilizando um outro instrumento para avaliar a depressão, Micco e Ehrenreich (2009) encontraram associações positivas e significativas com os fatores ameaça social $(r=0,41)$ e fracasso pessoal $(r=0,49)$. Todo esse conjunto de resultados suporta a hipótese de que os pensamentos automáticos negativos estão relacionados com os sintomas depressivos.

Análises de regressão com os quatro fatores da EPA e o sexo do participante como preditores da depressão mostraram que o fator fracasso pessoal é a única variável independente significativa. Esse resultado pode ser comparado com o descrito por D'Alessandro e Burton (2006), que encontraram que a visão negativa do self era um preditor para a depressão em crianças e adolescentes, em detrimento da visão negativa do mundo e do futuro. Os achados referentes à importância do fator fracasso pessoal na predição da depressão também vão de encontro à noção de especificidade de conteúdo postulada por Beck (1967), onde cada transtorno psicológico possui um perfil cognitivo específico. Esse construto foi testado por Schniering e Rapee (2004b) em amostras clínicas e comunitárias de crianças e adolescentes com idade entre sete e 16 anos, com transtornos psicológicos distintos, encontrando pensamentos automáticos relacionados a fracasso pessoal como os mais fortes preditores de sintomas depressivos (Schniering \& Rapee, 2004b). Tais dados são similares aos resultados encontrados no presente estudo, o que sugere que, na depressão, a especificidade de conteúdo da cognição se relaciona a temas de derrota, privação, autodepreciação e desesperança (Dozois \& Beck, 2008). Já em um nível cognitivo estrutural, os temas que caracterizam a depressão se referem a esquemas autorreferentes maladaptativos ou crenças envolvendo rejeição interpessoal, abandono e desaprovação ou crenças sobre falhas nas próprias conquistas, perda de domínio ou dependência dos outros (Beck, 1995).

\section{Considerações finais}


A versão traduzida e adaptada da Children's Automatic Thoughts Scale (CATS), chamada de Escala de Pensamentos Automáticos para Crianças e Adolescentes (EPA), mostrou resultados iniciais de validades fatorial e convergente e de consistência interna adequados, indicando ser $\mathrm{O}$ instrumento promissor para o uso no Brasil. No entanto, são necessários ainda estudos que investiguem outros aspectos da EPA, como a sua estabilidade e sua discriminação com grupos clínicos. Uma crítica que pode ser feita à constituição da escala é a falta de itens que avaliem pensamentos positivos. Essa deficiência, já corrigida em outros instrumentos, está sendo revista no instrumento original e foi recentemente publicada (Hogendoorn e cols., 2010). É interessante destacar que é necessário, além da tradução e adaptação de escalas, o desenvolvimento de instrumentos. Desse modo, poderiam ser contempladas áreas ou situações mais contextualizadas para a realidade brasileira. A EPA é um instrumento de avaliação de pensamentos automáticos geral, não sendo relacionada a nenhum transtorno em específico, e servirá como ponto de partida para esse tipo de avaliação no Brasil, em virtude da carência de instrumentos nessa área.

\section{Referências}

Beck J. S. (2011). Cognitive behavior therapy: Basics and beyond. $2^{\mathrm{a}}$ ed. New York: Guilford.

Beck, A. T. (1967). Depression: clinical, experimental, and theoretical aspects. New York: Hoeber.

Beck, A. T., \& Alford, B. A. (2011). Depressão. Causas e tratamento. Porto Alegre: Artmed.

Beck, A. T., Rush, A. J., Shaw, B. F., \& Emery, G. (1997). Terapia cognitiva da depressão. Porto Alegre: Artmed.

Beck, J. S. (1995). Cognitive therapy: basics and beyond. New York: Guilford.

Cruvinel, M., Boruchovitch, E., \& Santos, A. A. A. (2008). Inventário de Depressão Infantil (CDI). Análise dos parâmetros psicométricos. Fractal: Revista de Psicologia, 20, 473-490.

D'Alessandro, D. U. \& Burton, K. B. (2006). Development and validation of the Dysfunctional Attitudes Scale for Children: Tests of Beck's cognitive diathesis-stress theory of depression, of its causal mediation component, and of developmental effects. Cognition Therapy Research, 30, 335-353.

Dobson, K. S., \& Dozois, D. J. A. (2006). Fundamentos históricos e filosóficos das terapias cognitivo-comportamentais. Em K. Dobson (Ed.). Manual de terapias cognitivo-comportamentais (pp. 1743). Porto Alegre: Artmed.
Dozois, D. J. A. \& Beck A. T. (2008). Cognitive Schemas, beliefs and assumptions. Em K. S. Dobson \& D. J. A. Dozois (Eds.). Risk factors in depression (pp. 121-144). Oxford, England: Elservier/Academic Press.

Gabbard, G. O., Beck, J. S., \& Holmes, J. (2007). Compêndio de psicoterapia de Oxford. Porto Alegre: Artmed.

Gouveia, V., Barbosa, G., Almeida, H., \& Gaião, A. (1995). Inventário de Depressão Infantil - CDI: estudo de adaptação com escolares de João Pessoa. Jornal Brasileiro de Psiquiatria, 44, 345-349.

Hogendoorn, S. M., Wolters, L. H., Vervoort, L., Prins, P. J. M., Boer, F., Kooij, E., \& Haan, E. (2010). Measuring negative and positive thoughts in children: an adaptation of the Children's Automatic Thoughts Scale (CATS). Cognitive Therapy and Research, 34, 467-478

Hollon, S. D., \& Kendall, P. C. (1980). Cognitive self statements in depression: development of an Automatic Thoughts Questionnaire. Cognitive Therapy and Research, 4, 383-395.

Ingram, R. E., \& Wisnicki, K. S. (1988). Assessment of automatic positive cognition. Journal of Consulting and Clinical Psychology, 56, 898-902.

Ingram, R. E., Kendall, P. C., Siegle, G., Guarino, J., \& McLaughlin, S. C. (1995). Psychometric properties of the Positive Automatic Thoughts Questionnaire. Psychological Assessment, 7, 495-507

Kendall, P. C., Howard, B. L., \& Hays, R. C. (1989). Self-referent speech and psychopathology: the balance of positive and negative thinking. Cognitive Therapy and Research, 13, 583-598.

Kovacs, M. (1992). Children's Depression Inventory manual. Los Angels: Western, Psychological Services.

Kovacs, M. (2003). Children's Depression Inventory (CDI): technical manual update. Toronto: Multhi-Health Systems Inc.

Micco, J. A., \& Ehrenreich, J. T. (2009). Validity and specificity of the Children's Automatic Thoughts Scale in clinically anxious and non-clinical children. Cognitive Therapy and Research, 33, 532-536.

Ronan, K. R., Kendall, P. C., \& Rowe, M. (1994). Negative affectivity in children: development and validation of a self-statement questionnaire. Cognitive Therapy and Research, 18, 509-528.

Rutter, M. (1996). Stress research: accomplishments and tasks ahead. Em R. J. Haggerty, L. R. Sherrod, N. Garmezy, \& M. Rutter (Eds.). Stress, risk, and resilience in children and adolescents: process, mechanisms, and interventions (pp. 355-385). Cambridge: Cambridge University Press.

Schniering, C. A., \& Lyneham, H. J. (2007). The Children's Automatic Thoughts Scale in a clinical 
sample: psychometric properties and clinical utility. Behaviour Research and Therapy, 45, 19311940.

Schniering, C. A., \& Rapee, R. M. (2002). Development and validation of a measure of children's automatic thoughts: the Children's Automatic Thoughts Scale. Behaviour Research and Therapy, 40, 1091-1109.

Schniering, C. A., \& Rapee, R. M. (2004a). The relationship between automatic thoughts and negative emotions in children and adolescents: a test of the cognitive content-specificity hypothesis. Journal of Abnormal Psychology, 113, 464-470.

Schniering, C. A., \& Rapee, R. M. (2004b). The structure of negative self-statements in children and adolescents: a confirmatory factor analytic approach. Journal of Abnormal Child Psychology, 32, 95-109.
Schniering, C. A., \& Lyneham, H. J. (2007). The Children's Automatic Thoughts Scale in a clinical sample: psychometric properties and clinical utility. Behaviour Research and Therapy, 45, 19311940.

Wathier, J. L., Dell'Aglio, D. D., \& Bandeira, D. R. (2008). Análise fatorial do Inventário de Depressão Infantil (CDI) em amostra de jovens brasileiros. Avaliação Psicológica, 7, 75-84.

Zatz, S., \& Chassin, L. (1983). Cognitions of testanxious children. Journal of Consulting and Clinical Psychology, 51, 526-534.

Recebido em: 15/02/2012

Reformulado em: 19/03/2013

Aprovado em: 29/03/2013

Sobre os autores:

Maycoln L. M. Teodoro é professor adjunto do Programa de Pós-Graduação em Psicologia da Universidade Federal de Minas Gerais, e doutor pela A. L. Universität Freiburg (Alemanha) com pós-doutorado na UFRGS. Bolsista de Produtividade em Pesquisa do CNPq (2), é pesquisador mineiro pela FAPEMIG.

Heitor Amâncio de Moraes Castro é graduando em psicologia (UFMG), bolsista de iniciação científica (CNPq) do Laboratório de Processos Cognitivos.

Aline Abreu e Andrade é doutoranda em Psicologia do Desenvolvimento (UFMG), mestre em Psicologia do Desenvolvimento (UFMG) e pós-graduada em Terapia Comportamental (PUC-MG). Possui graduação em Psicologia (UFMG) e Formação em Terapias Cognitivas (IMTC).

Contado com o autor:

Universidade Federal de Minas Gerais

E-mail: mlmteodoro@hotmail.com

Houve a inclusão dos nomes dos demais autores, assim como o acréscimo de suas informações curriculares.

Publicado:

Maycoln Teodoro - Universidade Federal de Minas Gerais, Minas Gerais, Brasil

Correto:

Maycoln Teodoro - Universidade Federal de Minas Gerais, Minas Gerais, Brasil

Aline Abreu e Andrade - Universidade Federal de Minas Gerais, Minas Gerais, Brasil

Heitor Amâncio de Moraes Castro - Universidade Federal de Minas Gerais, Minas Gerais, Brasil 
